\title{
Old Wine in (Somewhat Leaky) New Bottles: Some Comments on Beall
}

August 30, 2016

\begin{abstract}
Dialetheists concerning the paradoxes of self-refrence have often argued that the phenomeonon provides a choice between inconsistency and expressive incompleteness, and that inconsistency is the correct choice. In a recent paper (Beall (2015)), JC Beall attacks this argument. This paper analyses his arguments, and argues that his paper simply provides a new spin on matters well known.
\end{abstract}

\section{Introduction}

Dialetheists about the semantic paradoxes of self-reference have often argued that one can have consistency or expressive completeness, but not both; and that inconsistency is better than incompleteness (since natural language obviously can express the notions in question). In 'Trivialising Sentences and the Promise of Semantic Completeness', ${ }^{1}$ JC Beall criticises this argument. He mounts an argument to the effect that one can have triviality or expressive completeness, but not both. Here, he argues, expressive incompleteness is the obviously correct choice. Moreover, he claims, since this argument is exactly parallel to the dialetheist argument, it should be treated in the same way. Hence, in the dialetheic case, we ought to accept incompleteness too. The point of this note is to comment on this argument. ${ }^{2}$

\footnotetext{
${ }^{1}$ Beall (2015). Page references are to this unless otherwise stated

${ }^{2}$ Many thanks go to Beall for a helpful correspondence, which clarified a number of matters in his paper, and to a referee of this journal for helpful comments.
}

Australasian Journal of Logic (13:5) 2016, Article no. 1 


\section{Curry Paradoxes}

The argument for the dialetheic conclusion is essentially the Liar paradox. This hardly needs an explanation here. So let us move to its supposed analogue, which Beall dubs the trivialiser paradox. To understand what is going on in this, it is important to put it in context. This is Curry's paradox. Let us start by recalling some facts about this.

The first version of Curry's paradox was produced by Curry (1942), and goes essentially as follows. We construct a sentence, $C$, of the form $C \rightarrow \perp$ (where $\perp$ is a logical constant entailing everything). We also assume the principle of Absorption: $A \rightarrow(A \rightarrow B) \vdash A \rightarrow B$, which contracts two occurrences of an antecedent into one. The argument now goes as follows:

$$
\begin{aligned}
& C \rightarrow C \\
& C \rightarrow(C \rightarrow \perp) \\
& C \rightarrow \perp \\
& C \\
& \perp
\end{aligned}
$$

The third line is delivered by Absorption. The other lines are self-explanatory.

In (1979) Meyer, Routley, and Dunn produced a variation of Curry's paradox which uses, not Absorption, but Pseudo Modus Ponens (PMP): $(A \wedge(A \rightarrow B)) \rightarrow B$. The argument goes as follows:

$$
\begin{aligned}
& (C \wedge(C \rightarrow \perp)) \rightarrow \perp \\
& (C \wedge C) \rightarrow \perp \\
& C \rightarrow \perp \\
& C \\
& \perp
\end{aligned}
$$

The third line obviously assumes some simple properties of conjunction. The rest of the argument is straightforward.

Though the argument looks rather different from the original Curry version, it is not really. The reason is that, in conjunction with some other natural principles, PMP is equivalent to Absorption. The proof in one direction is as follows:

$$
(A \wedge(A \rightarrow B)) \rightarrow B
$$

So if $A \rightarrow(A \wedge(A \rightarrow B))$ then $A \rightarrow B$

But $A \rightarrow A$

So if $\quad A \rightarrow(A \rightarrow B) \quad$ then $\quad A \rightarrow(A \wedge(A \rightarrow B))$

So if $A \rightarrow(A \rightarrow B) \quad$ then $A \rightarrow B$

Australasian Journal of Logic (13:5) 2016, Article no. 1 
The proof in the other direction is as follows:

$$
\begin{array}{ll} 
& ((A \rightarrow B) \wedge A) \rightarrow A \\
\text { So } & (A \rightarrow B) \rightarrow((A \rightarrow B) \wedge A) \rightarrow B) \\
\text { But } & ((A \rightarrow B) \wedge A) \rightarrow(A \rightarrow B) \\
\text { So } & ((A \rightarrow B) \wedge A) \rightarrow(((A \rightarrow B) \wedge A) \rightarrow B) \\
\text { So } & ((A \rightarrow B) \wedge A) \rightarrow B
\end{array}
$$

The last line is given by Absorption. Note that if we do not have this, we have only: $((A \rightarrow B) \wedge A) \rightarrow(((A \rightarrow B) \wedge A) \rightarrow B)$.

\section{Validity Curry}

In (2013), Beall and Murzi came up with a version of Curry's paradox, which uses, not the conditional, but a validity predicate, $\operatorname{Val}(x, y)$, which satisfies two rules. The first is $\mathrm{V} 1$ :

$$
\begin{gathered}
\bar{A} \\
\vdots \\
B \\
\operatorname{Val}(\langle A\rangle,\langle B\rangle)
\end{gathered}
$$

where angle brackets indicate naming, overlining indicates the discharge of an assumption, and there are no other assumptions in the sub-deduction. ${ }^{3}$ The second rule is V2:

$$
\frac{A \operatorname{Val}(\langle A\rangle,\langle B\rangle)}{B}
$$

Note that these are almost exactly the same as the rules of $\rightarrow$-introduction and $\rightarrow$-elimination. That they should give rise to a paradox of the same kind as the original Curry argument is hardly, therefore, surprising.

We now construct a sentence, $C$, of the form $\operatorname{Val}(\langle C\rangle,\langle\perp\rangle)$, and the

\footnotetext{
${ }^{3}$ For Beall, deducibility, and so $\mathrm{Val}$, are relative to a theory, T. This means that the deduction here may employ members of $T$ taken as discharged assumptions - or simply proofs thereof, if $T$ is an axiomatic theory.
}

Australasian Journal of Logic (13:5) 2016, Article no. 1 
argument goes essentially as follows:

$$
\begin{aligned}
& C \vdash C \\
& C \vdash \operatorname{Val}(\langle C\rangle,\langle\perp\rangle) \\
& C \vdash C \\
& C, C \vdash \perp \\
& C \vdash \perp \\
& \operatorname{Val}(\langle C\rangle,\langle\perp\rangle) \\
& C \\
& \perp
\end{aligned}
$$

The fourth line follows from the second and third by an application of V2; the sixth follows from V1. The rest is self-explanatory.

As a casual comparison will show, there is a clear structural similarity between this argument and the original version of Curry's Paradox. The contraction of conditional antecedents has just been turned into a contraction of assumptions.

Now, Beall's current paradox goes as follows. $A$ is a trivialiser iff $A \vdash \perp$; and a trivialiser predicate is a predicate, $\operatorname{Triv}(x)$, that says that something is a trivialiser. We can, in fact, define Triv using $\operatorname{Val}$, as follows: $\operatorname{Val}(x,\langle\perp\rangle)$. He now assumes the following:

(*) $A \wedge \operatorname{Val}(\langle A\rangle,\langle\perp\rangle) \vdash \perp$

Where $C$ is as before, we then argue as follows:

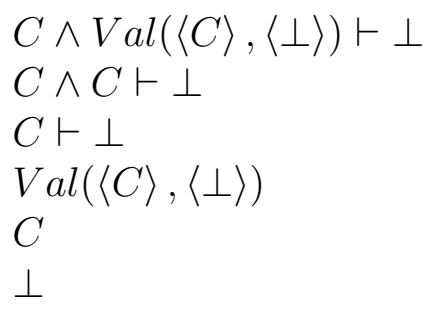

As a casual comparison will show, there is a clear structural similarity between this and the Meyer/Routley/Dunn version of Curry's Paradox.

As we saw when we discussed this, the use of PMP encodes a contraction of antecedents. So does $(*)$. There is nothing special about $\perp$ in this: $\left(^{*}\right)$ is just a special case of:

$(* *) A \wedge \operatorname{Val}(\langle A\rangle,\langle B\rangle) \vdash B$

Australasian Journal of Logic (13:5) 2016, Article no. 1 
Now, rewriting V2 as $A, \operatorname{Val}(\langle A\rangle,\langle B\rangle) \vdash B$, we can see that this delivers (*) provided that assumptions are allowed to contract, as follows:

$$
\begin{aligned}
& A \wedge \operatorname{Val}(\langle A\rangle,\langle B\rangle) \vdash A \\
& A \wedge \operatorname{Val}(\langle A\rangle,\langle B\rangle) \vdash \operatorname{Val}(\langle A\rangle,\langle B\rangle) \\
\text { So } \quad & \wedge \operatorname{Val}(\langle A\rangle,\langle B\rangle), A \wedge \operatorname{Val}(\langle A\rangle,\langle B\rangle) \vdash B \\
& A \wedge \operatorname{Val}(\langle A\rangle,\langle B\rangle) \vdash B
\end{aligned}
$$

The third line is delivered by V2. Just as with the Meyer/Routley/Dunn version of the Curry argument, without contraction, we have only the principle with two occurrences of the antecedent/assumption.

In fact, as can now be seen, Beall's argument is just the Meyer/Routley/Dunn version of the Validity Curry paradox. We still have the argument to deal with. But now it is clear that Beall's claim that the Liar and the Trivialiser are of the same kind is just the claim that the Liar and Curry's paradox are of the same kind. This is certainly a vexed question. But it is not a new one. $^{4}$

\section{The "Chief Challenge"}

In the last section of his paper, somewhat in tension with the stated aim of the paper, Beall says (p. 581, his italics):

The question - and the chief challenge of this paper - is to give a simple and plausible account of why the natural and highly plausible narrow-adequacy condition [GP: i.e., $(*)]$ should be rejected.

As we have seen, what is at issue here is precisely contraction, and what is being asked for is a justification of the failure of this. Premise-contraction is perfectly legitimate provided that assumptions are thought of as forming a set (or more generally, as Beall assumes, that a theory is a set). So what is required is a justification for the rejection of this.

In contraction-free substructural logics, such as linear logic and many relevant logics, one must distinguish between extensional conjunction, $\wedge$,

\footnotetext{
${ }^{4}$ Prima facie, the fact that contraction of some kind seems to play a role in all versions of the Curry Paradox, whilst it appears to play no role in the Liar, would seem to set them apart. But everything in this area is contentious. See, e.g., Weber, Ripley, Priest, Hyde, and Colyvan, (2014), Beall (2014), and Priest (201+), Section 15.
}

Australasian Journal of Logic (13:5) 2016, Article no. 1 
and fusion, o. When one does so, one does not have $(A \wedge(A \rightarrow B)) \rightarrow B$. However, one does have $((A \rightarrow B) \circ A) \rightarrow B$. (Arguably, it is the confusion of $\wedge$ and $\circ$ that motivates those who endorse PMP; and similarly, for the confusion of $A \wedge \operatorname{Val}(\langle A\rangle,\langle\perp\rangle) \vdash \perp$ with $A \circ \operatorname{Val}(\langle A\rangle,\langle\perp\rangle) \vdash \perp$.) In setting up a sequent calculus for such a logic, it is natural to deploy two ways of combining premises, $\oplus$ and $\otimes$ - corresponding to $\wedge$ and $\circ$, respectively. ${ }^{5}$ As is to be expected, we do not then have $A \oplus(A \rightarrow B) \vdash B$, but we have $(A \rightarrow B) \otimes A \vdash B$.

Given this machinery, premises of an inference do not form a set; they form what is sometimes called a bunch, that is, a formula obtained by interating operations of $\oplus$ and $\otimes$. We may, if we wish, then specify a theory by specifying a set of axioms, but this has to be understood as meaning that we are entitled to any bunch formed from those axioms. At any rate, this provides the justification Beall requests. ${ }^{6}$

Of course, one might challenge the whole machinery of sub-structural logics, and insist on an independent justification for that. There is, of course, an enormous literture on substructural logics, a good part of it directed to questions of justification. ${ }^{7}$ Here is not the place to go into this. It suffices to say that substructural logicians are well aware of Beall's challenge. So there is nothing really new in this matter either.

\section{References}

[1] Beall, J. (2014), 'The End of Inclosure', Mind 123: 829-49.

[2] Beall, J. (2015), 'Trivialising Sentences and the Promise of Semantic Completeness', Analysis 75: 573-84.

[3] Beall, J., and Murzi, J. (2013), 'Two Flavors of Curry's Paradox', Journal of Philosophy, 110: 143-165.

\footnotetext{
${ }^{5}$ Though, I note, some versions of substructural logic make use of only the intensional combinator.

${ }^{6}$ This is all explained in Priest (2015). Beall cites this paper and says that it (p. 581) 'does not, as far as I can see, clearly motivate a rejection of' $(*)$. He appears to have missed the point.

${ }^{7}$ For an entry into the literature, see Restall (2000) and Paoli (2002), and, for a survey, Restall (2008).
}

Australasian Journal of Logic (13:5) 2016, Article no. 1 
[4] Curry, H. (1942), 'The Inconsistency of Certain Formal Logics', Journal of Symbolic Logic 7: 115-17.

[5] Meyer, R., Routley, R. and Dunn, J. M. (1979) 'Curry's Paradox', Analysis 39: 124-8.

[6] Paoli, F. (2002), Substructural Logics: A Primer, Dordrecht: Kluwer.

[7] Priest, G. (2015), 'Fusion and Confusion', Topoi 34: 55-61.

[8] Priest, G. (201+), 'What If: the Exploration of an Idea', to appear.

[9] Restall, G. (2000), An Introduction to Substructural Logics, London: Routledge.

[10] Restall, G. (2008), 'Substructural Logics', in E. Zalta (ed.), Stanford Encyclopedia of Philosophy, http://plato.stanford.edu/entries/logicsubstructural/.

[11] Weber, Z., Ripley, D., Priest, G., Hyde, D., and Colyvan, M. (2014), 'Tolerating Gluts', Mind 123: 813-28.

Australasian Journal of Logic (13:5) 2016, Article no. 1 